\title{
Reflets
}

Revue ontaroise d'intervention sociale et communautaire

\section{La santé communautaire en Ontario français : défis et espoirs}

\section{Michèle Kérisit et Madeleine Dubois}

Volume 1, numéro 2, automne 1995

La santé communautaire en Ontario français : défis et espoirs

URI : https://id.erudit.org/iderudit/026074ar

DOI : https://doi.org/10.7202/026074ar

Aller au sommaire du numéro

Éditeur(s)

Reflets : Revue ontaroise d'intervention sociale et communautaire

ISSN

1203-4576 (imprimé)

1712-8498 (numérique)

Découvrir la revue

Citer ce document

Kérisit, M. \& Dubois, M. (1995). La santé communautaire en Ontario français : défis et espoirs. Reflets, 1(2), 9-15. https://doi.org/10.7202/026074ar

Tous droits réservés (C) Reflets : Revue ontaroise d'intervention sociale et communautaire, 1995
Ce document est protégé par la loi sur le droit d'auteur. L'utilisation des services d'Érudit (y compris la reproduction) est assujettie à sa politique d'utilisation que vous pouvez consulter en ligne.

https://apropos.erudit.org/fr/usagers/politique-dutilisation/ 


\section{La santé communautaire en Ontario français: défis et espoirs}

\section{M ichèle $K$ érisit et M adeleine $D$ ubois}

œ- rédactrices

D euxième livrai son de $R$ eflets, deuxième thème livré à la réflexion des intervenantes, intervenants, chercheures et chercheurs travaillant dans le domaine de l'intervention sociale et communautaire en 0 ntario français... Ce numéro porte sur l'histoire, les développements et la situation de la santé communautaire dans nos régions et au sein de nos collectivités. 0 ù en sommes-nous? Q uels sont les projets déjà en place? Q ue préparons-nous pour I'avenir de nos communautés? Q uels défis nous attendent? Telles sont les questions qui ont guidé notre travail durant les six derniers mois.

Le défi était de taille. D'une part, nous nous embarquions dans un domaine qui a été peu exploré en 0 ntario français. S'il existe certains ouvrages sur la question, ils sont souvent dispersés et ont rarement fait l'objet d'analyses systématiques. Existe-t-il une possibilité de dire la spécificité des pratiques en 0 ntario français, alors que la réflexion semble simplement débuter? D'autre part, le terme lui-même de santé communautaire ne fait pas nécessairement unanimité et embrasse un champ d'investigation tellement large que l'on peut se demander parfoiss'il ne s'agit pas de tout dire sur tout. Comment allions-nous décider de ce qui appartenait à ce thème et ce qui pourrait faire l'objet d'un numéro suivant, sur la violence ou sur le vieillissement, par exemple? Enfin, alors que nous préparions déjà ce numéro, l'élection d'un nouveau gouvernement en 0 ntario semblait requestionner certaines 
directions prises par le gouvernement précédent... Allions- nous arriver au mois de novembre avec une problématique périmée?

La lecture des articles, à la lumière des défis que nous avons voulu relever, nous porte à croire que ce deuxième numéro de $R$ eflets permettra aux lecteurs et aux lectrices de se faire une meilleure idée de la spécificité des pratiques de santé communautaire en 0 ntario français, de s'orienter dans le labyrinthe des pratiques de santé que l'on appelle communautaire et enfin d'explorer davantage l'importance, pour les Franco-O ntariens, des nouvelles tendances en matière de politiques de santé qui, justement, en appellent à la communauté.

II importe de souligner que, si nous avons tenté de faire le point sur la question de la santé communautaire, nous ne prétendons pas avoir fait un tour exhaustif de tous les projets et de tous les programmes qui existent dans la province. II existe sans doute d'autres expériences et pratiques importantes et pertinentes qui pourront, nous le souhaitons, être décrites et analysées dans les prochains numéros. Ajoutons que les pratiques de santé communautaire sont présentement en pleine évolution, non seulement en raison des contraintes budgétaires actuelles, mais aussi sous la poussée d'un mouvement qui se fait sentir dans l'ensemble du C anada et même dans le monde entier. L'O ntario français n'échappe pas à ce mouvement qui met l'accent sur des pratiques de santé plus proches des préoccupations quotidiennes des citoyens et veut réduire la part grandissante de la technologie dans les soins au profit d'une vision intégrée et politique de l'être humain en santé. C 'est dans cet esprit qu'il nous a semblé intéressant de publier une réflexion faisant état de l'expérience de Franco- $M$ anitobaines atteintes du cancer du sein. En bref, nous voulons souligner la nature exploratoire et la modestie de notre projet pour ce numéro de R eflets: il s'agissait de prendre une photographie, aussi précise que possible mais nécessairement partielle, à un moment dans l'évolution de l'O ntario français, des préoccupations, des défis et des espoirs en matière de santé. Q ue nous révèle donc ce portrait?

La lecture des articles, quelle que soit la rubrique où ilss'insèrent, nous révèle que les projets ou les pratiques franco-ontariennes 
s'inscrivent dans une histoire spécifique et indiquent leslieux où se sont développées, souvent silencieusement, des façons de faire répondant aux besoins particuliers de la population. $D$ ans leur travail sur la santé des travailleurs de quarts, Barette, Picard, B eyers et Taillefer illustrent l'importance des questions de santé en milieu industriel et minier, lieux intimement liés à l'histoire et à la réalité actuelle de nombreux Franco- $\mathrm{O}$ ntariens dans le $\mathrm{N}$ ord de la province. Adam, dans son analyse comparative des groupes d'entraide, démontre combien les variables linguistique et culturelle jouent dans le type de groupe existant dans la région de $M$ anitoulin-Sudbury et l'importance encore grande de la paroisse comme schème organisateur de l'appui et du réseautage dans la communauté francophone. L'entraide est d'ailleurs l'un des thèmes récurrents de ce recueil, partiellement en raison du sujet même (santé communautaire) qui valorise les prises en charge par l'entraide, mais aussi parce qu'elle repose sur des habitudes de vie et de survie propres aux communautés minorisées. U ne autre composante du tableau, le rôle des femmes dans les affaires de santé, traverse plusieurs articles recueillis pour ce numéro (C oderre; Lafrenière et $D$ enis; $R$ ail et $D$ allaire, $M$ alubungi; Paquette). Si ce rôle est actuellement en train de se développer et de s'affirmer, comme le dit Coderre, il n'en reste pas moins qu'il a été joué depuis bien longtemps, en particulier dans le domaine des soins aux personnes vivant avec des maladies chroniques, de la maternité et de l'enfantement et du soin aux enfants.

$N$ otonscependant que nousn'avons reçu aucun article portant sur l'histoire et les traditions populaires concernant la santé (pharmacopée traditionnelle reposant sur la connaissance des herbes et des plantes, organisations traditionnelles au sein des paroisses). II existe comme un grand silence, en 0 ntario français, sur les modes d'organisation et de savoir traditionnels de la santé communautaire. Est-ce par manque de documentation? Est-ce le fait d'avoir eu toujours à combattre pour pouvoir se faire une place au soleil, et donc ne pas vouloir révéler une non-modernité suspecte aux yeux de la société dominante? Est-ce aussi parce que les batailles de l'O ntario francophone se sont déroulées majoritairement dans le domaine de l'éducation, éclipsant du 
même fait d'autres préoccupations? Le texte de $R$ ail et $D$ allaire, qui rend compte de leur recherche-action sur les déterminants de la santé pour les Francophones de l'O ntario, mentionne comme déterminant important l'accèsà une éducation de qualité en français. II nous apparaît en effet essentiel de tisser desliens plusétroits entre les acquis résultant des revendications dans le domaine de l'éducation et les préoccupations naissantes concernant la santé.

La spécificité actuelle des pratiques en santé communautaire se remarque également dans certains thèmes communs qui émergent des articles. $M$ algré leur diversité régionale et leurs objectifs variés, de nombreux programmes valorisent la participation des usagers et des usagères dans l'expression de leurs besoins. L'article de Lemonde et Pomerleau sonde les besoins en santé mentale des groupes de personnes âgées dans la région de Sudbury; le Centre de santé communautaire de la même ville procède d'une étude des besoins de la population (Lafrenière et D enis); la création de Franc- 0 -Forme (Théberge) insiste sur la reconnaissance des besoins spécifiques de se maintenir en forme en français.

Parallèlement, nous avons remarqué une volonté de mettre l'accent sur la participation active et la prise en charge des programmes par les usagers eux-mêmes. Les différents acteurs de l'intervention s'engagent dans la mobilisation des usagers et usagères pour la création de nouvelles formes de services de santé, en français. C ette insistance sur l'importance de la voix des usagers et usagères, de la spécificité de leurs besoins et de leur expérience, est importante si l'O ntario francophone veut se doter d'un réseau de santé communautaire digne de ce nom. Ici, en effet, on voit se dessiner l'émergence d'un mouvement de santé communautaire qui ne veut plus attendre que lui soit octroyée la permission de se construire dans sa spécificité linguistique et culturelle. $C$ 'est ce que montre Blais, à travers une analyse des modèles explicatifs dominants en santé mentale. Son article illustre combien ces modèles explicatifs dominants ont pu et peuvent toujours stigmatiser les personnes pauvres lorsqu'elles mettent pauvreté et problèmes de santé mentale dans le même panier. En adhérant plus ou moins inconsciemment à un appareil de 
recherche et d'intervention qui ne laisse aucune place à ce qu'elle appelle des réal ités autres, nous sommes en passe de faire de l'image d'une personne aisée et blanche une norme de santé, sans prendre en considération la manière dont les facteurs socio-culturels présidant à l'émergence des problèmes de santé mentale sont «représentés, vécus et exprimés dans des contextes spécifiques et différents». Labrèche le dit autrement dans ses poèmes qui émaillent ce numéro de $\mathrm{R}$ eflets.

U n autre thème qui prédomine est celui de l'importance des liens sociaux dans le maintien et l'amélioration de la santé dans les communautés francophones de I'O ntario. Les liens sociaux créés dans les cuisines collectives de H earst (G uindon), les solidarités et les amitiés qui naissent dans l'Épicerie-bus du centre ville d'O ttawa (Larochelle), I'effort collectif que représente l'autoformation proposée par la C oalition franco- ontarienne sur le logement (Bergeron) et l'organisation de solidarités dansle $\mathrm{N}$ ord- ouest ontarien (Paquette) font voir à quel point la santé ne se réduit plus à la visite chez le médecin. Elle a aussi partie liée à une action collective qui déboucheà la foissur un mieux-être individuel et sur celui des communautés (D upont; $N$ arbonne-Fortin et collègues).

Finalement nous voulons souligner l'importance des articles portant sur la santé des femmes et sur leurs rôles comme dispensatrices de soins ou comme usagères du système. Ils nous montrent à quel point, longtemps silencieuses, les pratiques de santé communautaire sont encore conduites par les femmes ontaroises. Par leur travail d'entraide, par les modes d'organisation qu'elles se sont donnés et par leur questionnement des pratiques médicales institutionnelles dominantes (C oderre), elles sont, peut-être, celles qui oeuvrent actuellement le plus en faveur de l'établissement d'un réseau de santé communautaire. $\mathrm{N}$ ées en O ntario ou venues d'ailleurs, les femmes ont toutes pour souci le maintien des liens sociaux comme garants d'une possibilité de meilleure santé et une volonté de changer les systèmes dominants qui définissent le bien-être et la santé des femmes en termes souvent inacceptables pour elles. 
C ertains paramètres se dégagent d'ores et déjà des pratiques de santé communautaire en 0 ntario français: spécificité linguistique et culturelle des besoins et du vécu des usagers et usagères et lutte pour la reconnaissance de cette spécificité, importance des liens entre intervenantes et intervenants et la communauté, importance desfemmeset de leurs organismes pour mettre en oeuvre un certain nombre de programmes. II nous semble, cependant, que nous sommes ici face à une naissance, à une éclosion, plutôt qu'à une mise en oeuvre concertée. De nombreux obstacles se dressent devant la réalisation de certains projets, comme le signale Wesley R omulus, directeur du C entre médico-social communautaire de Toronto, dans une entrevue qu'il a accordée à $R$ eflets en mai dernier. Passons les en revue, rapidement, maisfermement.

Le contexte actuel dans lequel évolue le réseau socio-sanitaire ontarien dansson ensemble semble aller dansle sens d'un développement de plus en plus décentralisé, désinstitutionnalisé et communautaire du réseau. Les hôpitaux sont sur la sellette, les cliniques communautaires sont de plus en plus reconnues comme partenaires importants dans la livraison des services de santé. B ref, la santé communautaire est à la mode... Chance à saisir, certainement, mais qui ne va pas sans danger.

O n peut, en effet, se demander si la force d'application de la Loi 8 pourra résister à cette décentralisation des services et à l'importance accrue des conseils régionaux de santé. Q uelle est la part que prendront les Francophones dans cette restructuration des services, en particulier dans la répartition des fonds? $\mathrm{N}$ ous sommes nous donné les outils nécessaires à une participation active dans les instances décisionnelles? Pouvons- nousfaire en sorte que le travail accompli (et toujours à recommencer) pour l'éducation soit aussi fait pour les questions de santé? En tant que rédactrices nous nous attendions à quel ques considérations sur la question dans ce numéro. C ertains des textes y font rapidement allusion... mais nous n'avons trouvé personne pour examiner le problème à fond. La question reste donc posée...

La soi-disant dévolution des pouvoirs de décision aux niveaux communautaires et régionaux a certes pour conséquence de 
favoriser certains organismes communautaires. On peut cependant se demander s'ils seront en mesure de répondre aux demandes multipliées résultant du réajustement des services et comment nous nous préparons à cet avenir assez rapproché. II existe actuellement des services de santé communautaire florissants en anglais. La communauté francophone sera-t-elle en mesure de répondre à la demande des siens ou va-t-elle voir ses clientèles potentielles se tourner vers les services en anglais pour des raisons bien connues de proximité, mais aussi parce que les solutions de rechange, desalternatives au système institutionnel comme celles que présente $\mathrm{C}$ arole Labrèche, n'auront pas été mises en place? $\mathrm{N}$ ous nous devons d'amorcer une réflexion de fond sur ce que signifie la santé mentale et physique - et lesmeilleurs moyens pour la maintenir et l'améliorer - pour les Franco- 0 ntariens et les Franco- $O$ ntariennes. Si nous pouvons en déceler les prémisses dans certains démarches rapportées dans ce numéro de $R$ eflets, I'on peut se demander si elles constituent une base suffisante pour affirmer notre présence et notre réalité face à des instances politiques pour lesquelles seule prime une rationalité économique à court terme.

N ous pourrions certes multiplier les questions d'o rdre politique à l'infini... N ous ne pouvons que souligner leur importance, et la nécessité urgente d'une réflexion collective à ce sujet. 